\title{
The gears of the Antikythera Mechanism: an educational pathfinder to the solar system
}

Xenophon Moussas ${ }^{1}$, Georgios Bampasidis ${ }^{1}$, Yanis Bitsakis ${ }^{2}$, Theodosios Tassios ${ }^{3}$, Magdalini Anastasiou ${ }^{4}$, Kyriakos Efstathiou ${ }^{5}$, Georgios Fasoulopoulos $^{6}$, Isidoros Kioleoglou ${ }^{7}$, Mike Edmunds ${ }^{8}$, Mary Zafeiropoulou $^{9}$, Manos Roumeliotis ${ }^{10}$, Tom Malzbender ${ }^{11}$, Andrew

Ramsey ${ }^{12}$, Dionysios Kriaris ${ }^{13}$, Evangelos Spandagos ${ }^{14}$, Nikos Giannopoulos ${ }^{15}$, Amalia Porligi ${ }^{15}$, Eleni Daniels ${ }^{16}$, Michael T. Wright $^{17}$, Reem Sabry ${ }^{18}$, Hoda El-Mikaty ${ }^{18}$, Göran Henriksson ${ }^{19}$, Ing-Marie Munktell ${ }^{20}$, Flora Vafea ${ }^{21}$, Stratos Koufos ${ }^{22}$, Dimitrios Prassopoulos $^{23}$, Vasili Zafiropoulos ${ }^{24}$, Andreas Karakonstantis ${ }^{25}$, Katerina Aggeioplasti ${ }^{23}$, Eleni Delidou ${ }^{23}$, Costas Papoulias ${ }^{26}$, George Papoulias $^{26}$, Paul Haley ${ }^{27}$, Jamal Mimouni ${ }^{28}$, David Valls-Gabaud ${ }^{29}$, Matt Biggs ${ }^{27}$, Drahomir Chochol ${ }^{30}$, Jacek Szubiakowski ${ }^{31}$, Ela Plucinska $^{31}$, Anna Jacyno ${ }^{31}$, Alexandros Kakouris ${ }^{1}$, Pavel Pal'uš ${ }^{32}$, Danica Jančušková ${ }^{33}$, Mária Feriančiková ${ }^{32}$, Krystof Czart ${ }^{31}$, Nikos Xenakis $^{34}$, Magda Evgenia Gkini ${ }^{29}$, Eva Perpyraki ${ }^{35}$, Leonidas Tziannoudakis ${ }^{36}$, and Panagiotis Antoniou ${ }^{23,37}$

${ }^{1}$ Dept. of Astrophysics, Astronomy \& Mechanics,

Faculty of Physics, National \& Kapodistrian University of Athens,

Panepistimiopolis, 15783 Zographos, Athens, Greece email: xmoussas@phys.uoa.gr gbabasid@phys.uoa.gr

${ }^{2}$ University of Athens, Athens Science and Education Laboratory, Athens, Greece

${ }^{3}$ Association for Ancient Greek Technology Studies, Athens, Greece

${ }^{4}$ Faculty of Physics, Aristotle University of Thessaloniki, Greece

${ }^{5}$ Dept. of Mechanical Engineering, Aristotle University of Thessaloniki, Greece

${ }^{6}$ Secondary Education, Athens, Greece

${ }^{7}$ Ionic Center, 11 Lysiou Street, 10556, Athens, Greece

${ }^{8}$ Cardiff University, School of Physics \& Astronomy, Queens Buildings, Cardiff CF243AA, UK

${ }^{9}$ National Archaeological Museum of Athens, 1 Tositsa Str., 10682 Athens, Greece

${ }^{10}$ Department of Technology Management, University of Macedonia, Thessaloniki, Greece

${ }^{11}$ Hewlett-Packard Laboratories, Palo Alto, California, USA

${ }^{12}$ X-Tek Systems, Metris, UK ${ }^{13}$ Lysiou 2, GR 11146 Galatsi, Athens, Greece

${ }^{14}$ Aethra Publishing Company, Messolongiou 1, Athens, Greece ${ }^{15}$ Digital Artists, Athens, Grece

${ }^{16}$ DanielsMedia Co., Public Relations, NY, USA

${ }^{17}$ Centre for the History of Science, Technology \& Medicine, Imperial College, London, UK

${ }^{18}$ Planetarium Science Center, Bibliotheca Alexandrina, Alexploratorium, Alexandria, Egypt

${ }^{19}$ Division of Astronomy and Space Physics, Uppsala University, 75120 Uppsala, Sweden

${ }^{20}$ Gustavianum Museum, Uppsala University, Akademigatan 3, 75310 Uppsala, Sweden

${ }^{21}$ Abet Greek School in Cairo, Egypt

${ }^{22}$ Eudemus Observatory, Rhodes, Greece

${ }^{23}$ Amateur Astronomers Club of Thrace, 5 J. Betsou, 68100 Alexandropolis, Greece

${ }^{24}$ Faculty of Physics, University of Patras, Greece ${ }^{25}$ EKFE, Chios, Greece

${ }^{26}$ Solonos 112, Athens, Greece

${ }^{27}$ The SHARE Initiative, UK

${ }^{28}$ Department of Physics, University of Constantine, Algeria

${ }^{29}$ Observatoire de Paris, 5 Place Jules Janssen, 92195 Meudon, France ${ }^{30}$ Astronomical 


\author{
Institute of the Slovak Academy of Sciences, \\ Tatranska Lomnica, Slovakia \\ ${ }^{31}$ Olsztyn Planetarium and Astronomical Observatory, \\ Al. Piłsudskiego 38, 10-450 Olsztyn, Poland \\ ${ }^{32}$ Comenius University Bratislava, Slovakia \\ ${ }^{33}$ Centre for Astronomy of N. Copernicus University, \\ Gagarina 11, 87-100 Toruń, Poland \\ Prešov Planetarium, Slovakia \\ ${ }^{34}$ Kasos, Greece \\ ${ }^{35}$ Rethymon, Crete, Greece \\ ${ }^{36}$ EKFE, Rethymon, Crete, Greece \\ ${ }^{37}$ Democritus University of Thrace, Greece
}

\begin{abstract}
The Antikythera Mechanism is the most sophisticated extant ancient astronomical instrument and analogue computer known and was assembled sometime between 150 and 100 BCE, almost a century after the death of Archimedes. The mechanism has a great educational potential as it appeals to inquiring minds as an astonishing artefact of science and technology. The latest research findings reveal significant cultural and social functions in its operations. This astonishing astronomical instrument has a clear interdisciplinary valueand it has that it may be used as an educational medium, to engage the general public, and especially to attract students both to/from exact sciences and to/from the humanities. The astronomical and technical knowledge embedded in the mechanism can also be used to introduce some aspects of modern science through the unknown technological achievements of Hellenic antiquity.
\end{abstract}

Keywords. Antikythera Mechanism, astronomical instruments, education, modelling, outreach.

\title{
1. Introduction
}

The Antikythera Mechanism is an astonishing astronomical instrument of the ancient Hellenic world. The unknown craftsman constructed it with gears made of bronze to carry out astronomical calculations. The construction made up of gears with carefully-designed transmission ratios makes possible to perform the computations in the rational numbers domain. The instrument probably epitomises all the astronomical knowledge of its era. The Mechanism is a unique artefact in many ways -and the only device of its kind known today. It is both an astronomical instrument (Rediadis 1903) and an analogue computer (Price 1959, 1974; Theofanidis 1934). Scientists and engineers understood its priceless value since the day of its discovery (Svoronos 1903).

It was discovered in 1901-2 near the small Greek island of Antikythera during a sponge dive (Svoronos 1903). The fragments were part of the cargo of a large shipwreck of the 1st century BCE that was full of treasures been transported from Greece to Rome. This intriguing and mysterious device has perplexed many scientists who have been studying it through today (Moussas 1991; Freeth et al. 2006, 2008; Wright 2007). The research studies of the Mechanism continually unveil extraordinary information about the ancient Greek science and technology, some of which are unknown and very surprising. It is an instrument whose study leads to a complete revision of the history of science, technology, astronomy, mathematics and even philosophy.

The ancient instrument of the Mechanism has many dials with circular and two spiral displays, with pointers, some of which are quite complex. One dial is on the "front" and the rest of the dials on the "back" side of the Mechanism. The pointers are driven by gears that rotate by a single input shaft (Freeth et al. 2006, 2008; Price 1959, 1974; Theofanidis 1934). The device was originally housed in a wooden case, with its manual 
written on every available surface of the Mechanism. It appears to have been constructed between 150 and 100 BCE (Charis Kritzas, 2006, private communication).

The bronze fragments of the original are on display at the National Archaeological Museum of Athens and they are being globally investigated, like other important items of astronomical instruments.

\section{The interdisciplinary value of the Antikythera Mechanism}

As technology penetrates into the global community's daily routine, people find themselves in need of different types of technology applications and gadgets that they have to use every day. As a result, they need to develop special skills and acquire appropriate knowledge in order to understand the way things work and avoid the possibility of looking at them as mere offsprings of magic. Despite its mysterious and complex nature, the Antikythera Mechanism is a unique educational resource and tool. It is suitable for teaching modeling of nature by using laws of nature, mathematical methods and the translation of the gears' motions into mathematics. The Mechanism is the oldest known mechanical device in the history of civilisation, which proves that science can model the universe with mathematics, astronomy and engineering.

The Mechanism is a great attractor to children for science, mathematics and technology, but also for astronomy, philosophy, history, linguistics, geography, modeling, physics, metallurgy and many more (Moussas et al. 2007). Therefore, exhibitions around the world (see Table 1 below) highlighting the Mechanism, offer a tremendous opportunity for interaction between scientists and the public. For example, the Children's Museum of Manhattan (CMOM) in New York City is the only museum in America currently showcasing a reconstruction model of the Mechanism. Also, through their short audiovisual presentation visitors, from age 6 to adults, learn that it was the ancient Greek understanding of causality that led to the development of the scientific method we use today. At a nearby interactive kiosk, visitors learn how modern technology is being used to unlock the mysteries of the Antikythera Mechanism and learn how this Greek machine is altering our understanding of the history of science (Roumeliotis 1999).

These versatile events focus on the display of an exact replica of the Antikythera Mechanism in the same scale as the original. The reconstruction models depict the Mechanism's geared infrastructure and reveal its brilliant operation. Apart from the reconstructed device, each exhibition includes multimedia interactive software that enables the visitor to watch the history of the Mechanism as well as investigate and understand its functions and capabilities. In addition, elucidatory panels are on display at the surrounding area, in order to involve the visitor in the roots of science and astronomy. During the exhibitions, public lectures concerning the amazing story of the Mechanism are given to the general public, introducing ancient technology. In turn, this allows visitors of all ages to easily view the conjunction of modern science and historical heritage through the Antikythera Mechanism -and continue the conversation at home! Most importantly, these exhibitions constitute a dynamic way for studying and learning more about astronomy, mathematics and science, revealing the interdisciplinary value of the Antikythera Mechanism.

The Antikythera Mechanism is an incredibly resourceful and educational tool for changing the way the general public views Science and assists people to approach astronomy, mathematics, science and philosophy.

\section{Acknowledgements}

We thank the Ioannis F. Costopoulos Foundation, Leverhulme Trust, the University of Athens, the Aristotle University of Thessaloniki, The National Archaeological Museum 
Table 1. Overview of exhibitions of the Antikythera Mechanism.

\begin{tabular}{|c|c|c|c|}
\hline Event & Place & Date & Visitors \\
\hline National touring exhibition & Hellenic Museum, Chicago, USA & 2010 & \\
\hline $\begin{array}{l}\text { Gods, Myths and Mortals: Discover Ancient } \\
\text { Greece, Children's Museum of Manhattan }\end{array}$ & New York, USA & $2007-2010$ & 500,000 \\
\hline Aurora Polaris, Grundtvig & Olsztyn Planetarium, Poland & May-Sep 2009 & 10,000 \\
\hline University of Patras Center & Patras, Greece & Mar 2009 & 2,000 \\
\hline Culture Center City of Rehtymnon & Rehtymnon, Greece & 27-30 Mar 2009 & 300 \\
\hline Museum Gustavianum, Uppsala & Uppsala, Sweden & 31 Jan-29 Apr 2009 & 10,000 \\
\hline $\begin{array}{l}\text { Inauguration of IYA } 2009+\text { IAU Sympo- } \\
\text { sium } 260\end{array}$ & UNESCO, Paris, France & 15-23 Jan 2009 & 2,500 \\
\hline $\begin{array}{l}\text { Planetarium Science Center Bibliotheca } \\
\text { Alexandrina exploratorium }\end{array}$ & Alexandria, Egypt & $1-30$ Nov 2008 & 2,000 \\
\hline $\begin{array}{l}\text { Zappeion, Research and Innovation expo- } \\
\text { sition }\end{array}$ & Athens, Greece & Nov 2008 & 3,000 \\
\hline Abet Greek School in Cairo & Cairo, Egypt & 29 Nov 2008 & 300 \\
\hline Exhibition at CRAAG & Algiers Observatory, Algeria & 2 Nov 2008 & 50 \\
\hline 7ème Salon d'Astronomie & Constantine, Algeria & 30 Oct-1 Nov 2008 & 6,000 \\
\hline Ionic Centre & Athens, Greece & 22 Oct-14 Dec 2008 & 7,000 \\
\hline HELEXPO/DETH Intnl Fair & Thessaloniki, Greece & Sep 2008 & 4,000 \\
\hline Aurora Polaris, Grundtvig & Athens, Greece & Sep 2008 & 30 \\
\hline Church Children Camp N. Makri & Alexandroupolis, Greece & 1 Aug 2008 & 140 \\
\hline Amphitheatre of Gymnasium & Kasos, Greece & $30 \mathrm{Jul} 2008$ & 300 \\
\hline Karditsa Cultural Center & Karditsa, Greece & 28 Feb 2008 & 300 \\
\hline City of Chios & Chios, Greece & 9 Feb 2008 & 200 \\
\hline City of Ermioni & Ermioni, Greece & Feb 2008 & 120 \\
\hline HELEXPO/DETH Intnl Fair & Thessaloniki, Greece & Sep 2007 & 4,000 \\
\hline Municipal Theatre & Alexandroupolis, Greece & 5 May 2007 & 180 \\
\hline $\begin{array}{l}\text { Zappeion, Research and Innovation expo- } \\
\text { sition }\end{array}$ & Athens, Greece & May 2007 & 3,000 \\
\hline
\end{tabular}

of Athens, HP, X-tek Systems (METRIS), Volume Graphics GmbH, the Greek Ministry of Culture and UNESCO for supporting this research and exhibitions.

\section{References}

Freeth, T., Bitsakis, Y., Moussas, X., Seiradakis, J.H., Tselikas, A., Mangou, H., Zafeiropoulou, M., Hadland, R., Bate, D., Ramsey, A., Allen, M., Crawley, A., Hockley, P., Malzbender, T., Gelb, D., Ambrisco, W. \& Edmunds, M.G. 2006, Nature, 444, 587

Freeth, T., Jones, A., Steele, J.M. \& Bitsakis, Y. 2008, Nature, 454, 614

Moussas, X. 1991, in Hellenic Educational Encyclopedia, Vol. 14 (Athens: Ekdotiki Athinon)

Moussas, X., Seiradakis, J., Freeth, T., Edmunds, M., Bitsakis, Y., Babasides, G., IoannidisVamvakas, D., Fasoulopoulos, G., Daniels, E. \& Kriaris, D. 2007, in Communicating Astronomy to the Public, IAU Commission 55 conference 2007 (Athens) http://www. communicatingastronomy.org/cap2007/abstracts.html

Price, D.J. De Solla 1959, Scientific American, 200, 60

Price, D.J. de Solla 1974, Transactions of the American Philosophical Society (New Series), 64, part 7,1

Rediadis, P. 1903, Der Astrolabos von Antikythera (Athens: Beck \& Barth)

Roumeliotis, M. 1999, The Antikythera Mechanism, Multimedia CD-ROM, (Thessaloniki: Technological Museum of Thessaloniki)

Svoronos, J.N. 1903, Die Funde von Antikythera (Athens: Beck \& Barth)

Theofanidis, J. 1934, Praktika tes Akademias Athcnon, 9, 140

Wright, M.T. 2007, Interdisciplinary Science Reviews 32, 27 